\title{
Poesia, giustizia e carcere: dall' Inferno di Dante alla Città Dolente
}

\author{
Lorenzo Bartoli \\ Universidad Autónoma de Madrid \\ lorenzo.bartoli@uam.es
}

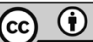

\section{Riassunto}

Il saggio esamina lo spettacolo teatrale Dalla Città Dolente: colpa, pena e liberazione nelle visioni dell'Inferno di Dante, scritto e diretto da Fabio Cavalli, con la compagnia del Teatro Libero di Rebibbia, mettendo in evidenza come l'incontro tra la poesia dell'Inferno e l'universo degli attori-detenuti inviti a riflettere sui concetti di colpa, giustizia retributiva e giustizia riparativa, in Dante e nella società contemporanea.

Parole chiave: Dante; Cavalli; teatro; carcere; colpa.

\begin{abstract}
The essay examines the dramatic piece Dalla Città Dolente: colpa, pena e liberazione nelle visioni dell'Inferno di Dante, written and directed by Fabio Cavalli, with the theatre company of the Teatro Libero di Rebibbia, emphasizing how the encounter of Inferno's poetry with the existencial universe of the prisoners-actors encourages a reflection on the concepts of guilt, retributive justice and reparative justice in Danteand in contemporary society.
\end{abstract}

Keywords: Dante; Cavalli; theatre; prison; guilt. 
T a recente traduzione italiana della monografia di Justine Steinberg su 1 Dante e il diritto (Steinberg 20r6); nonché la pubblicazione di vari studi di carattere storico e filologico sui documenti concernenti il bando di Dante del 1302 (Milani 20II), hanno riportato il tema della giustizia al centro del dibattito critico dantesco e particolarmente dell'Inferno, a cominciare dalla struttura carceraria che lo articola e del sistema giuridico che la amministra.

La messa in scena di Dalla Città Dolente. Colpa, Pena e Liberazione attraverso le visioni dell'Inferno di Dante con drammaturgia e regia di Fabio Cavalli con la compagnia del Teatro Libero del carcere romano di Rebibbia, costituisce un notevole study case per elaborare questa linea di ricerca, nella direzione di un confronto dialettico - attorno al concetto di giustizia - fra la poesia dantesca, da una parte, e le istanze di rinnovamento più urgenti legate al sitema carcerario ed alla società contemporanea, più in generale, dall'altra (Cavalli 20I5). ${ }^{\mathrm{I}}$

La compagnia Gi2 A.S del Teatro Libero di Rebibbia, nota per aver interpretato il film dei fratelli Taviani Cesare deve morire vincitore dell'orso d'argento a Berlino nel 20I2, e di cinque David di Donatello, è composta dagli attori-detenuti del braccio di Alta Sicurezza della Casa Circondariale romana di Rebibbia. Nata nel 2003 e diretta da Fabio Cavalli, ha messo in scena, nell'auditorio del centro di Rebibba, numerosi spettacoli teatrali tratti da opere di Shakespeare (Tempesta, Amleto, Giulio Cesare), Giordano Bruno, Eduardo De Filippo, Tolstoj, Bertolt Brecht. Fra questi, lo spettacolo su cui ci soffermiamo, ovvero Dalla Città Dolente. Colpa, Pena e Liberazione attraverso le visioni dell'Inferno di Dante, con drammaturgia e regia dallo stesso Fabio Cavalli.

Si tratta di uno spettacolo di circa un'ora e dieci minuti, incentrato sulla poesia dell'Inferno di Dante, rivissuta attraverso l'esperienza e la lingua degli attori-detenuti. In questo senso, lo spettacolo riunisce due filoni importantissimi della tradizione dantesca, ovvero quella della resa dialettale, da una parte, della lingua di Dante; e quella della drammatizzazione del poema. Ma l'aspetto più notevole del lavoro di Cavalli riguarda la sovrapposizione dell'universo poetico dell' Inferno con l'esperienza umana dei detenuti di un carcere, e ancor più dei detenuti reclusi in un braccio di Alta Sicurezza, soggetti a particolari restrizioni di regime carcerario.

Suddiviso in 9 scene e un'introduzione (una struttura, quindi, di reminiscenza dantesca), lo spettacolo vede, sin dall'inizio, gli attori-detenuti presenti sulla scena. Accompagnati dal pianista Franco Morelli, essi si alternano sul proscenio per rispondere o per essere interrogati, come se fossero a scuola o

I. Lo spettacolo - che in occasione della Festa del cinema di Roma (20I6) è andato in mondovisione, attraverso una proiezione in streaming dal MAXXI di Roma - si può vedere in Vimeo in Full-HD: https://vimeo.com/user548545I6/videos. 
a un quiz televisivo: Scena I: I golosi; Scena 2: La politica; Scena 3: Peccati, peccatori e fessi; Scena 4: Pietas; Scena 5: Ugolino; Scena 6: Ulisse; Scena 7: I traditori; Scena 8: Gli iracondi; Scena 9: I lussuriosi.

Il carcere come un inferno, i detenuti come dannati. Lo spettacolo e il testo della Città Dolente devono una parte importante della loro forza drammatica alla riflessione sul rapporto fra la condizione carceraria degli attori e della messa in scena all'interno del proprio carcere, e la condizione delle anime dei dannati nell'Inferno di Dante, nonché della propria esperienza biografica di Dante come esiliato, come latitante (Cavalli 2015):

Noi che viviamo qui dentro, per parlare della nostra condizione spesso chiamiamo in causa l'immagine di un inferno [...] la privazione della libertà, la vita reclusa, la riflessione sulle proprie responsabilità... sono causa di un dolore che è difficile da esprimere. Per questo facciamo teatro: per cercare di esprimere il senso di questo dolore attravreso le parole di chi ha la fantasia, l'immaginazione, la sensibilità per farlo; i grandi poeti. Quando poi il poeta è Dante - un condannato, un esiliato, uno che ha passato metà della sua vita da latitante - quando è Dante a parlarne, bisogna ascoltare e riflettere. Per questo siamo qua. Ciascuno di noi col suo Dante personale, con i versi scovati nella biblioteca del carcere, versi difficili, all'inizio misteriosi. Poi, piano piano, giorno dopo giorno, versi compresi, apprezzati, amati, perché - che ve lo dico a ffa? - Dante, se lo conosci, te ne innamori. Magari tradotto e un po' storpiato in lingue e dialetti diversi, come avete sentito all'inizio: spagnolo, napoletano, e poi sentirete il calabrese. Ma cosa importa? Sono convinto che Dante non si offende. Cominciamo! A ciascuno il suo peccato, a ciascuno il suo canto!

La rifunzionalizzazione del testo dantesco come strumento di presa di coscienza sulla propria condizione carceraria avviene innanzitutto sul piano formale, attraverso la resa dialettale di alcuni degli episodi più noti dell'Inferno. La versione spagnola di Bartolomé Mitre; il calabrese di Salvatore Scervini o il napoletano di Matilde Pierro Donnarumma, conferiscono allo spettacolo un senso di avvicinamento sentimentale alla lezione poetica dantesca, di urgenza personale. Così, l'episodio di Ulisse (Scena 6) diviene, nella drammaturgia di Cavalli e, nella messa in scena della compagnia del Teatro Libero di Rebibbia, l'occasione per ricordare e rivivere la sicilianità degli attori-detenuti che lo interpretano, avvicinandosi, in questo senso, al Dante di Primo Levi in Se questo è un uomo, dove il ricordo delle montagne piemontesi si sovrapponeva alla ricostruzione memoriale dei versi con cui si descrive, nella Commedia (If. XXVI), la montagna del Purgatorio (Levi 1958 e in particolare il capitolo XI dedicato a Il canto di Ullisse).

Il plurilinguismo della Città Dolente, quindi, oltre a recuperare un elemento determinante del dettato poetico dell'Inferno e della sua tradizione (Contini 1970; Arcangeli 2016), esprime la pregnanza esistenziale che i versi della Commedia proiettano sull'esperienza umana degli attori-detenuti: a ciascuno il 
suo peccato, a ciascuno il suo canto. A ciascuno la sua lingua. La drammaticità che scaturisce da questa lettura dell' Inferno si manifesta nel modo più forte e più toccante all'altezza dell'episodio di Paolo e Francesca, laddove i versi di Dante ("Nessun maggior dolore/che ricordarsi del tempo felice/ ne la miseria", If. $\mathrm{V}$, I2I-I23) diventano occasione per misurare, nella proibizione all'amore, la durezza della condizione carceraria (Cavalli 20I5):

Questi versi del V Canto li voglio dire perché, parlando del carcere, bisogna dirli per forza. Perché sembra che parlino di una storia d'amore antica, e invece parlano soprattutto della nostra vita, qui dentro, dove l'amore è proibito. E quando noi incontriamo le nostre donne, le nostre mogli, al parlatoio, per un'ora la settimana, e ci stringiamo le mani, uno di qua e l'altra di là dal muro, ciascuno è Paolo e ciascuna è Francesca. Sono le parole più belle, più vere, più terribili per descrivere cosa prova un essere umano quando l'amore lo può solo ricordare, e rimpiange, giorno dopo giorno, nel silenzio del suo inferno.

Altrettanto significativo dello scambio che interviene fra il testo dantesco e l'esperienza degli attori-detenuti, e particolarmente tenendo in considerazione che la compagnia del Gi2 AS riunisce attori detenuti nel braccio di alta sicurezza del carcere, appare la Scena 7 , nella quale il peccato di tradimento, di infamità, come si riformula nel testo di Cavalli, emerge a indicare ciò che risulta inaccettabile nella prospettiva del codice carcerario, sicché gli stessi attori deposti a discuterne si rifiutano non solo di parlarne ma persino di nominarlo (Cavalli 2015):

- Scusate questo teatrino, ma a noi c'è toccato un argomento che tutti avevano scartato. Hanno approfittato che noi due avevamo l'influenza e si sono presi gli argomenti più facili: la gola, l'ira, l'avarizia, la lussuria... a noi ci hanno lasciato il peccato più difficile. Soprattutto per uno che sta qua dentro... Ti dispiace dire qualcosa pure tu?

- Che cosa vuoi che dica?

- Il nome del peccato.

- Ah no! Io sono cattolico! Non mi va.

- Nemmeno a me.

- Allora statti zitto.

- Allora statti zitto pure tu.

- Buonasore, allora parlo io. Il tradimento. Il peccato che non vogliono dire è il tradimento. Qui nessuno ne vuole parlare, perché è un argomento scottante.Perché noi lo chiamiamo con un'altra parola: infamità. Perché molti, qua dentro, ci sono finiti in questo modo: per il tradimento di qualcuno che ha parlato. Ha fatto l'infame. ${ }^{2}$

2. Sull'importanza del concetto di infamia nell'universo giuridico-poetico di Dante, nonché nella cultura figurativa medievale, oltre al già citato volume di Justin Steinberg (Steinberg 20I6, in particolare il cap. I: Beneath the Law: Infamia, pp. 13-52) si vedano gli studi di Gherardo Ortalli (Ortalli 1979) e Giuliano Milani (Milani 2008). Per il rapporto fra infamia e autobiografia in Dante (ma con osservazioni che ben potrebbero estendersi alla situazione dei 
Qui il testo di Cavalli raggiunge il suo momento forse più importante dal punto di vista della denuncia sociale. Il problema del tradimento, infatti, non riguarda solamente la condanna dei detenuti, ma anche le specifiche condizioni di regime carcerario a cui essi sono sottoposti. Per i detenuti appartenenti al Gi2 di Rebibbia, infatti, soggetti in gran parte all'articolo 4Ibis del codice penitenziario, è esclusa qualsiasi forma di riduzione della pena, se non attraverso la collaborazione con la giustizia. Ovvero, secondo l'antropologia carceraria, attraverso il tradimento. Il percorso di riparazione dell'individuo si scontra quindi con la logica retributiva del sistema penale: la pena, a dispetto dell'articolo 27 della Costituzione italiana, rischia di diventare definitiva e irremissibile. $^{3}$

L'immagine del carcere come un inferno, quindi, non agisce solo come metafora, quantunque pregnante e carica di significato: diviene piuttosto il nucleo sentrale della riflessione metalinguistica degli attori-detenuti sul percorso intrapreso di presa di cocienza della propria condizione carceraria e di riparazione dell'individuo: attraverso il teatro, attraverso l'università, attraverso Dante. A dispetto del codice penitenziario, e della logica retributiva della giustizia, ciò che lo spettacolo di Cavalli viene a segnalare è dunque una strada diversa di riparazione, un diverso concetto di giustizia, dove la ferita sociale creata dal delitto non si ripara esclusivamente attraerso la resa dei conti penitenziaria, ma implica un complesso percorso di assunzione delle proprie responsabilità da parte del detenuto. Un percorso che, attuando una sorta di riumanizzazione del detenuto, aiuti anche la società stessa a recuperare un atteggiamento più umano verso il carcere che costituisce il senso stesso della giustizia di riparazione. È stato studiato, e ne parla Cavalli nel suo intervento su Dante e l'Arte, che il lavoro teatrale e di coinvolgimento dei detenuti in attività di rieducazione, favoriscono in modo notevole la riduzione del tasso di reincisività dei detenuti stessi: una volta scontata la pena, i detenuti che in carcere hanno svolto questo tipo di attività reincidono nel delitto in misura notevolmente minore rispetto al rsto dei detenuti. Ma l'importanza di questo tipo di interventi non riguarda solo il detenuto: riguarda piuttosto l'impegno civile della società verso una giustizia non solo retributiva ma anche riparativa: nella società contemporanea, come nell'Inferno di Dante.

detenuti.attori di Rebibbia ed alla loro urgenza autobiografica), si rimanda al saggio di Elisa Brilli (Brilli 20II).

3. Per un approfondimento sul tema del rapporto fra carcere, cultura e università, si veda il numero monografico di Mosaico, Una officina di riparazione dell'individuo: l'università (di Tor Vergata) in carcere, XIII.I46 (marzo 20I6), a cura di Fabio Pierangeli, Patricia Peterle e Andrea Santurbano (Santurbano, Pierangeli, Peterle 20I6), nel quale si pubblicano gli interventi al convegno, svoltosi presso l'auditorium della Casa Circondariale di Rebibbia il iz febbraio 20I6, sul tema: Il carcere: da Università del crimine a officina di riparazione della persona. Vari degli attori-detenuti del Teatro Libero di Rebibbia, nonché studenti dell'Università di Tor Vergata, hanno partecipato al convegno (Bonetti 20I6; Moscato 20I6; Falbo 20I6). 


\section{BIBLIOGRAFIA}

Alighieri, Dante, 1966, La Commedia: Secondo l'Antica Vulgata, a c. di G. Petrocchi, Milano, Mondadori.

—, I922, La Divina Comedia, traducida en verso por a Bartolomé Mitre, Buenos Aires, Latium.

Arcangeli, M., 2016, Dialetti senza frontiere. Le traduzioni della Commedia di Dante nelle varie "lingue" d'Italia, relazione in Poesia e Dialetto, a cura di C. Pulsoni, Perugia, I4 giugno 2016.

Bonetti, J. D., 20I6, "Il carcere e il processo di antropopoiesi", in Santurbano Pierangeli - Peterle, 20I6, pp. I2-I6.

Brilli, E., 20II, "L'arte di dire l'esilio", in Bollettino di Italianistica. Rivista di critica, storia letteraria, filologia e linguistica, VIII, 2, pp. 17-4I.

Cavalli, F., 20I5, Dalla Città Dolente. Colpa, pena e liberazione attraverso le visioni dell'Inferno di Dante, Roma, Edizioni La Ribalta, Centro Studi Enrico Maria Salerno.

Contini, G., I970, "Preliminari sulla lingua del Petrarca", in Id., Varianti e altra linguistica, Torino, Einaudi, pp. I69-92.

Falbo, F., 20I6, "Un doloroso problema mai affrontato", in Santurbano - Pierangeli Peterle, 2016, pp. 19-20.

Levi, P., I958, Se questo è un uomo, Torino, Einaudi.

Milani, Giuliano, 2008, "Prima del Buongoverno. Motivi politici e ideologia popolare nelle pitture del Broletto di Brescia", Studi medievali 49, pp. I9-85.

—, 20II, "Appunti per una riconsiderazione del bando di Dante", Bollettino di Italianistica. Rivista di critica, storia letteraria, filologia e linguistica, VIII, 2, pp. $42-70$.

Moscato, A. M., 2016, "Cultura e trasformazione del sé”, in Santurbano - Pierangeli Peterle, 2016, pp. I7-I8.

Ortalli, G., I979, La pittura infamante nei secoli XIII-XVI. Roma, Jouvence.

Santurbano, A., Pierangeli, F., Peterle, P. (ed.), 20I6, Mosaico, Una officina di riparazione dell'individuo: l'università (di Tor Vergata) in carcere, XIII.I46.

Steinberg, J., 20I6, Dante e $i$ confini del diritto, Roma, Viella (tit. orig. Dante and the Limits of the Law, University of Chicago Press, Chicago, 2013). 